Weakly bound states of two- and three-boson systems in the crossover from two to three dimensions

This content has been downloaded from IOPscience. Please scroll down to see the full text. 2015 J. Phys. B: At. Mol. Opt. Phys. 48025302

(http://iopscience.iop.org/0953-4075/48/2/025302)

View the table of contents for this issue, or go to the journal homepage for more

Download details:

IP Address: 186.217.236.152

This content was downloaded on 14/10/2015 at 20:39

Please note that terms and conditions apply. 


\title{
Weakly bound states of two- and three- boson systems in the crossover from two to three dimensions
}

\author{
M T Yamashita ${ }^{1}$, F F Bellotti ${ }^{2,3,4}$, T Frederico $^{2}$, D V Fedorov ${ }^{3}$, \\ A S Jensen ${ }^{3}$ and N T Zinner ${ }^{3}$ \\ ${ }^{1}$ Instituto de Física Teórica, UNESP_Univ Estadual Paulista, C.P. 70532-2, CEP 01156-970, São Paulo, \\ SP, Brazil \\ ${ }^{2}$ Instituto Tecnológico de Aeronáutica, 12228-900, São José dos Campos, SP, Brazil \\ ${ }^{3}$ Department of Physics and Astronomy, Aarhus University, DK-8000 Aarhus C, Denmark \\ ${ }^{4}$ Instituto de Fomento e Coordenação Industrial, 12228-901, São José dos Campos, SP, Brazil \\ E-mail: yamashita@ift.unesp.br
}

Received 12 August 2014, revised 23 October 2014

Accepted for publication 10 November 2014

Published 31 December 2014

\begin{abstract}
The spectrum and properties of quantum bound states is strongly dependent on the dimensionality of space. How this comes about and how one may theoretically and experimentally study the interpolation between different dimensions is a topic of great interest in different fields of physics. In this paper we study weakly bound states of non-relativistic two and three boson systems when passing continuously from a three (3D) to a two-dimensional (2D) regime within a 'squeezed dimension' model. We use periodic boundary conditions to derive a surprisingly simple form of the three-boson Schrödinger equation in momentum space that we solve numerically. Our results show a distinct dimensional crossover as three-boson states will either disappear into the continuum or merge with a 2D counterpart, and also a series of sharp transitions in the ratios of three-body and two-body energies from being purely $2 \mathrm{D}$ to purely $3 \mathrm{D}$.
\end{abstract}

Keywords: cold atoms, Efimov states, low-dimensional systems

\section{Introduction}

Strongly interacting quantum particles are of great importance in many fields including nuclear and particle physics, condensed-matter physics, and atomic and molecular physics. However, they can be very hard to approach due to their intrinsically non-perturbative nature and often it is necessary to employ numerical simulations such as Monte Carlo [1] and/or lattice field theory techniques as done with great success in lattice QCD [2]. In the limit of few particles, strongly interacting systems can display truly remarkable features such as the Efimov effect [3] where a geometric series of threebody bound states of three bosons occur at the threshold for the binding of any two-body subsystem. This is a key insight into the often counterintuitive behavior of few-body systems that is extremely difficult to capture without analytical guidance. Moreover, it is an effect that is intimately tied to the dimensionality of space as it will not happen in two dimensions.

Cold atomic gases have proven their ability as excellent quantum simulation tools due to the tunability of interactions, geometry, and inter-particle statistical properties [4-6]. A recent frontier is the study of strongly interacting atomic Bose gases in three [7-13] and most recently in two dimensions $[14,15]$. At the few-body level, three-body states linked to the Efimov effect have been observed in three dimensions [1636] using a variety of different atomic species and two-body Feshbach resonances [37]. In spite of the tunability of the external trapping geometry of cold atomic systems, there has been little study of how the three-boson bound state problem undergoes its dramatic change from displaying the Efimov effect in three dimensions to having only two bound states in two dimensions [38]. A key question is whether it is possible to interpolate these limits in simple theoretical terms and 
subsequently explore this in simulations using both more involved numerical methods and experimental setups.

Here we present a model that has the ability to interpolate geometrically between two and three spatial dimensions and thus study this important crossover for both two- and threebody bound states of identical bosons. A 'squeezed' dimension is employed with periodic boundary conditions (PBC) whose size can be varied to interpolate the two limits. It has the unique feature that it can be regularized analytically which is a great advantage for its numerical implementation allowing us to go smoothly between both limits. While PBC is not typically used in few-body calculations, it is nevertheless a standard trick when addressing larger systems [1, 39, 40] and our results may thus also serve as a benchmark. Since the seminal work of Wilson [41] and t'Hooft [42], using the dimensionality of a given model as a parameter has been a standard tool in high-energy and condensed matter physics. More recently such techniques have been applied to strongly interacting Fermi gases [43] in the context of cold atoms. Moreover, in the realm of few-body physics mixed dimensional systems are promising for extension of the Efimov scenario to new setups [44].

For many experimental setups in cold atoms, the transverse confining geometry is given by a harmonic trapping potential and a very interesting recent theoretical study [45] has considered the properties of three-boson states under strong transverse confinement. The very recent successful production of box potential traps for bosons [46, 47] means that open (hard wall) boundary conditions (OBC) are now also accessible. This still leaves the question of how to realize $\mathrm{PBC}$ in a cold atomic gas experiment. However, we would not expect large qualitative differences between $\mathrm{OBC}$ and $\mathrm{PBC}$ and more likely only quantitative changes. This is of course also one of the reasons for employing PBC in most contexts throughout physics. The theoretical elegancy and tractability of calculations in the three-body system is the strong incentive that we have for pursuing this geometry even if at present an experimental realization has not been found.

\section{Dimer energy with PBC}

In our model we will assume PBC along one direction (chosen to be the $z$-axis) which is initially a condition on the single-particle coordinates. We will now argue, however, that it may be implemented in the relative momentum which significantly simplifies its implementation. Let $p_{1}$ and $p_{2}$ be the $z$-components of the momenta of particles 1 and 2 with coordinates $z_{1}$ and $z_{2}$, respectively. Then we may rewrite

$$
p_{1} z_{1}+p_{2} z_{2}=Z p_{\mathrm{CM}}+z p_{z}
$$

where $p_{\mathrm{CM}}=\left(p_{1}+p_{2}\right) / \sqrt{2}$ and $p_{z}=\left(p_{1}-p_{2}\right) / \sqrt{2}$, while $Z=\left(z_{1}+z_{2}\right) / \sqrt{2}$ and $z=\left(z_{1}-z_{2}\right) / \sqrt{2}$. PBC implies that we must quantize according to $p_{1}=2 \pi n_{1} / L$ and $p_{2}=2 \pi n_{2} / L$, where $L$ is the length of the periodic dimension. We thus see that $p_{\mathrm{CM}}$ and $p_{z}$ will in turn also be quantized as a sum or a difference of a pair of integers.
Furthermore, the Hamiltonian separates in $\mathrm{CM}$ and relative momenta. Thus we may treat $p_{\mathrm{CM}}$ and $p_{z}$ independently.

Disgarding the center-of-mass momentum we may now concentrate on the relative part. The relative momenta along the plane are then given by $\vec{p}_{\perp}=\left(p_{x}, p_{y}\right)$, while

$$
p_{z}=\frac{\sqrt{2} \pi n}{L}=\frac{n}{R},
$$

with $n=\left(n_{1}-n_{2}\right)=0, \pm 1, \pm 2 \ldots$ and $L=\sqrt{2} \pi R$. The length of the squeezed dimension corresponds to a radius, $R$, that interpolates between the two-dimensional (2D) limit for $R \rightarrow 0$ and the three-dimensional (3D) limit for $R \rightarrow \infty$. In this paper we will use a contact (zero-range) interaction to study the continuous transition from $3 \mathrm{D}$ to $2 \mathrm{D}$ regimes.

Here we consider the case where we allow $E_{2}$ to vary with $R$ under the physical condition that the magnetic field is fixed and thus we have a fixed dimer energy in $3 \mathrm{D}, E_{2}^{3 \mathrm{D}}$. This implies that the two-body T matrix in the limit of $R \rightarrow \infty$ has to recover a pole exactly at $E_{2}^{3 \mathrm{D}}$. This means that for finite $R$ we must solve

$$
\int \frac{\mathrm{d}^{3} p}{E_{2}^{3 \mathrm{D}}-p^{2}}-\frac{1}{R} \sum_{n} \int \frac{\mathrm{d}^{2} p_{\perp}}{E_{2}-p_{\perp}^{2}-\frac{n^{2}}{R^{2}}}=0 .
$$

As both terms in equation (3) are divergent, ultraviolet cutoffs that are consistent with the correct 3D limit must be introduced. It is enough to regularize the transverse momentum integral $\mathrm{d}^{2} p_{\perp}$ in both terms of (3) with a cutoff $\Lambda$ and then take the limit $\Lambda \rightarrow \infty$.

$$
\begin{gathered}
\lim _{\Lambda \rightarrow \infty}\left\{\int_{-\infty}^{\infty} \mathrm{d} y \ln \left[\frac{-E_{2}^{3 \mathrm{D}} R^{2}+y^{2}}{-E_{2}^{3 \mathrm{D}} R^{2}+y^{2}+(\Lambda R)^{2}}\right]\right. \\
\left.-\sum_{n=-\infty}^{\infty} \ln \left[\frac{E_{2} R^{2}-n^{2}}{E_{2} R^{2}-n^{2}-(\Lambda R)^{2}}\right]\right\}=0,
\end{gathered}
$$

where $y \equiv R p$. Performing the analytical integration and the sum we have

$$
\begin{aligned}
& \lim _{\Lambda \rightarrow \infty}\left\{\pi R\left(\sqrt{-E_{2}^{3 \mathrm{D}}}-\sqrt{-E_{2}^{3 \mathrm{D}}+\Lambda^{2}}\right)\right. \\
& \left.\quad-\ln \left(\frac{\sinh \pi \sqrt{-E_{2}} R}{\sinh \pi \sqrt{-E_{2}+\Lambda^{2}} R}\right)\right\} \\
& =\pi R \sqrt{-E_{2}^{3 \mathrm{D}}}-\ln \left(2 \sinh \pi \sqrt{-E_{2}} R\right)=0 .
\end{aligned}
$$

By recognizing that $\sqrt{-E_{2}^{3 \mathrm{D}}} \rightarrow 1 / a$ in the zero-range limit (where $a$ is the two-body scattering length), the energy of the dimer is

$$
\sqrt{-E_{2}}=\frac{1}{\pi R} \sinh ^{-1} \frac{\mathrm{e}^{\pi R / a}}{2} .
$$

The energy of the dimer is shown in figure 1 . For $R \rightarrow 0$ it goes to $\sqrt{-E_{2}} \sim(\pi R)^{-1} \sinh ^{-1} \frac{1}{2}=0.153174 R^{-1}$, which does not depend on the scattering length. Therefore, for any 3D two-body subsystem-bound or virtual-a strong deformation of the trap towards $2 \mathrm{D}$ always binds the dimer with an 


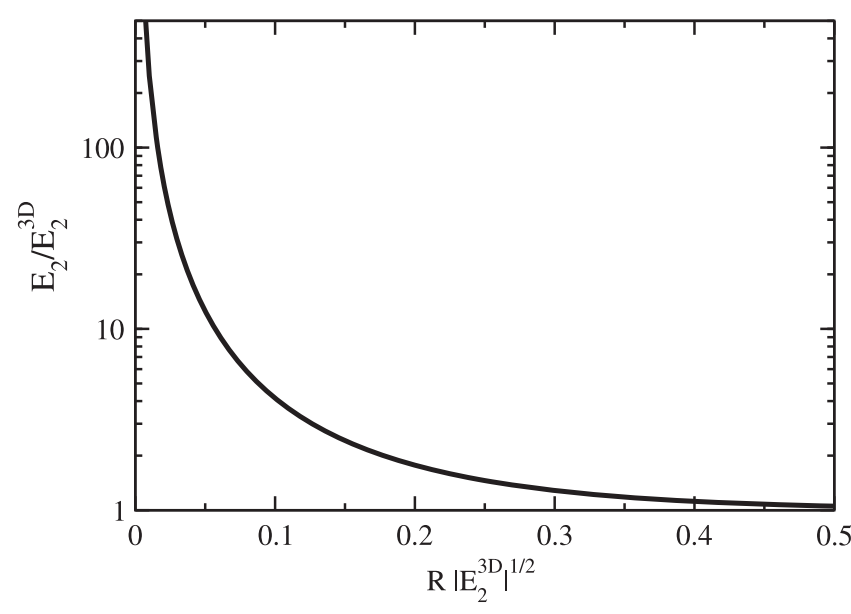

Figure 1. Dimer energies $E_{2} / E_{2}^{3 \mathrm{D}}$ as a function of $R \sqrt{\mid E_{2}^{3 \mathrm{D} \mid}}$ for periodic boundary conditions.

energy given by the trap energy scale $\left(1 / R^{2}\right.$ in this case). In the unitary limit where $a \rightarrow \infty$ we find this bound state energy for any finite $R$. This is analogous to the famous quasi2D harmonic trap result of Petrov and Shlyapnikov [54]. In general, the precise way in which a low-dimensional geometry is obtained will be reflected in the dimer energy formula. This is similar to Fermi gases in non-trivial confinement [55].

\section{Thomas-Efimov effect with a squeezed dimension}

The zero-range three-boson equation with a periodic dimension can be obtained as a generalization the Skorniakov and Ter-Martirosian (STM) equation [48]. For completeness, let us first establish the STM equation for a regular 3D system in homogeneous space (see [49] for a discussion of the general form of the STM equations in 3D).

$$
\begin{aligned}
f(\vec{q})= & 2 \tau_{3 \mathrm{D}}\left(E_{3}-\frac{3}{4} q^{2}\right) \\
& \times \int \mathrm{d}^{3} p \frac{f(\vec{p})}{E_{3}-q^{2}-p^{2}-\vec{q} \cdot \vec{p}},
\end{aligned}
$$

where $E_{3}$ is the three-body energy and $f(\vec{q})$ is the so-called spectator function which uniquely defines the three-body wave function [49]. As we only consider the equal mass case, there is only one spectator function (a detailed discussion can be found in [50]). Here $\tau_{3 \mathrm{D}}$ is the two-body T-matrix given below in equation (14).

We may now split the momentum variable in the directions transverse to $\left(p_{\perp}\right.$ and $\left.q_{\perp}\right)$ and parallel to $\left(p_{z}\right.$ and $\left.q_{z}\right)$ the 'squeezed' dimension. This means that along the $z$-axis the momenta have to be quantized as discussed above. The integral over all momenta in the STM equation thus becomes an integral over $p_{\perp}$ and a sum over the modes of the periodic dimension. Likewise, the kinetic energy (or free Green's function) is naturally split into continuous $\left(p_{\perp}\right)$ and discrete contributions $\left(p_{z}\right)$. The generalized STM equation now becomes

$$
\begin{aligned}
& f\left(\vec{q}_{\perp}, n\right)=2 \tau_{R}\left(E_{3}-\frac{3}{4}\left(q_{\perp}^{2}+\frac{n^{2}}{R^{2}}\right)\right) \\
& \times \sum_{m} \int \mathrm{d}^{2} p_{\perp} \frac{f\left(\vec{p}_{\perp}, m\right)}{E_{3}-q_{\perp}^{2}-p_{\perp}^{2}-\vec{q}_{\perp} \cdot \vec{p}_{\perp}-\frac{n^{2}}{R^{2}}-\frac{m^{2}}{R^{2}}-\frac{n m}{R^{2}}},
\end{aligned}
$$

where $\tau_{R}$ is the two-body T-matrix that we discuss momentarily (see equations (11) and (12)). The parameter that interpolates between two and three dimensions is $R$. When $R$ approaches zero we are effectively in $2 \mathrm{D}$ and in the opposite limit where $R \rightarrow \infty$ we go to the 3D case.

We now discuss the Thomas collapse [51] and Efimov effect [3]. In momentum space the matrix elements of the Dirac $\delta$-function potential are constant. This introduces a singularity which must be resolved by proper regularization [52] which introduces a subtraction in the kernel of the zerorange three-boson integral equation, such that the properly regularized STM equations become

$$
\begin{aligned}
& f\left(\vec{q}_{\perp}, n\right)=2 \tau_{R}\left(E_{3}-\frac{3}{4}\left(q_{\perp}^{2}+\frac{n^{2}}{R^{2}}\right)\right) \\
& \times \sum_{m} \int \frac{\mathrm{d}^{2} p_{\perp}}{R}\left[g_{0 R}(E)-g_{0 R}\left(-\mu^{2}\right)\right] f\left(\vec{p}_{\perp}, m\right),
\end{aligned}
$$

where

$$
\begin{aligned}
g_{0 R}^{-1}(E)= & E-q_{\perp}^{2}-p_{\perp}^{2}-\vec{q}_{\perp} \cdot \vec{p}_{\perp} \\
& -\frac{n^{2}}{R^{2}}-\frac{m^{2}}{R^{2}}+\frac{n m}{R^{2}} .
\end{aligned}
$$

Here $f\left(\vec{p}_{\perp}, m\right)$ is the momentum-space three-body wave function that we need to determine. The two-body T-matrix, $\tau_{R}$, is given by

$$
\begin{aligned}
R \tau_{R}^{-1}(E)= & \sum_{n} \int \frac{\mathrm{d}^{2} p_{\perp}}{E-p_{\perp}^{2}-\frac{n^{2}}{R^{2}}} \\
& -\sum_{n} \int \frac{\mathrm{d}^{2} p_{\perp}}{E_{2}-p_{\perp}^{2}-\frac{n^{2}}{R^{2}}}
\end{aligned}
$$

where $E<0$ and we chose the bound-state pole at $E_{2}$. Throughout most of this paper we will use units of $\hbar=\mu=M=1$, where $M$ is the boson mass and $\mu$ is the momentum-space subtraction point of the regularization procedure [52]. Performing the analytical integration over $\vec{p}_{\perp}$ and the sum, we have

$$
\tau_{R}(E)=-R\left[\pi \ln \left(\frac{\sinh \pi \sqrt{-E} R}{\sinh \pi \sqrt{-E_{2}} R}\right)\right]^{-1} .
$$

Taking into account that the T-matrix in $2 \mathrm{D}$ and $3 \mathrm{D}$ have 
different units, we recover the two limits via

$$
\tau_{2 \mathrm{D}}(E)=\lim _{R \rightarrow 0} R^{-1} \tau_{R}(E)=-\left[\pi \ln \left(\frac{\sqrt{-E}}{\sqrt{\left|E_{2}\right|}}\right)\right]^{-1},
$$

which reproduces the standard 2D amplitude [52], and for $R \rightarrow \infty$ we obtain

$$
\tau_{3 \mathrm{D}}(E)=\lim _{R \rightarrow \infty} \tau_{R}(E)=\frac{1}{\pi^{2}}\left[\sqrt{E_{2}}-\sqrt{-E}\right]^{-1},
$$

valid for $E<0$. For continuum energies, $E>0$, the analytical extension in (13) and (14) can be performed from negative to the positive energy through the upper half of the complex energy plane. There is a subtlety in equation (9) since for any $R$ the kernel is noncompact if the subtraction term is disregarded. Therefore, to take the $2 \mathrm{D}$ limit one has first to regularize equation (9) and then take the limit $R \rightarrow 0$. In addition, to get the famous $2 \mathrm{D}$ results of Bruch and Tjon [38], one has to also take the limit $E_{2} \rightarrow 0$.

For $R \rightarrow 0$, the Efimov limit given by $E_{2} \rightarrow 0$ disappears because the homogeneous equation (9) reduces to its usual 2D form. In this case, only states with $n=0$ are relevant. For higher $n$ the kinetic energy blows up and this makes terms with $n>0$ in the kernel of the bound state equation irrelevant. The 3D Thomas collapse (infinitely negative ground state three-body energy) occurs when letting $\mu \rightarrow \infty$ in equation (9) after taking $R \rightarrow \infty$. For any finite $R$, the threebody ground state still collapses for $\mu \rightarrow \infty$ as the kernel of equation (9) is noncompact if the subtraction term is ignored. Here we will work in the limit where $\mu$ is finite and we take units such that $\mu=1$ as discussed above. We note that the equivalence between the Thomas and Efimov effects that happens in 3D [53] is broken with a compact dimension.

\section{Trimer energy with PBC}

We now present the numerical solution of the trimer bound state equation in equation (9). The calculations presented are done for two different values of $E_{2}$ which are independent of $R$ for reason of numerical convenience. In an experiment this could be achieved by using a magnetic Feshbach resonance and changing the field along with the trap size in such a manner that $E_{2}$ remains fixed. We will address shortly what qualitative changes we expect for the case where $E_{2}$ varies with $R$ as we have discussed in the dimer section above. Introducing explicit units for clarity, the dimensionless quantities we will use are $\epsilon_{3}=E_{3} / E_{0}, \epsilon_{2}=E_{2} / E_{0}$ and $r=R \mu / \hbar$, where $E_{0}=-\frac{\hbar^{2} \mu^{2}}{M}$. In order to explore the dimensional crossover transition, figure 2 shows the ratios $\epsilon_{3} / \epsilon_{2}$ as a function of $r$ for the ground, first, and second excited states. Note that the last state goes into the continuum before the $2 \mathrm{D}$ limit is reached.

The points are calculated for two fixed two-body energies $\epsilon_{2}^{3 \mathrm{D}}=10^{-6}$ (empty circles/dashed lines) and $10^{-7}$ (full circles/ solid lines). In a pure 3D calculation these parameters are on the $a>0$ side of the resonance and one finds three three-body

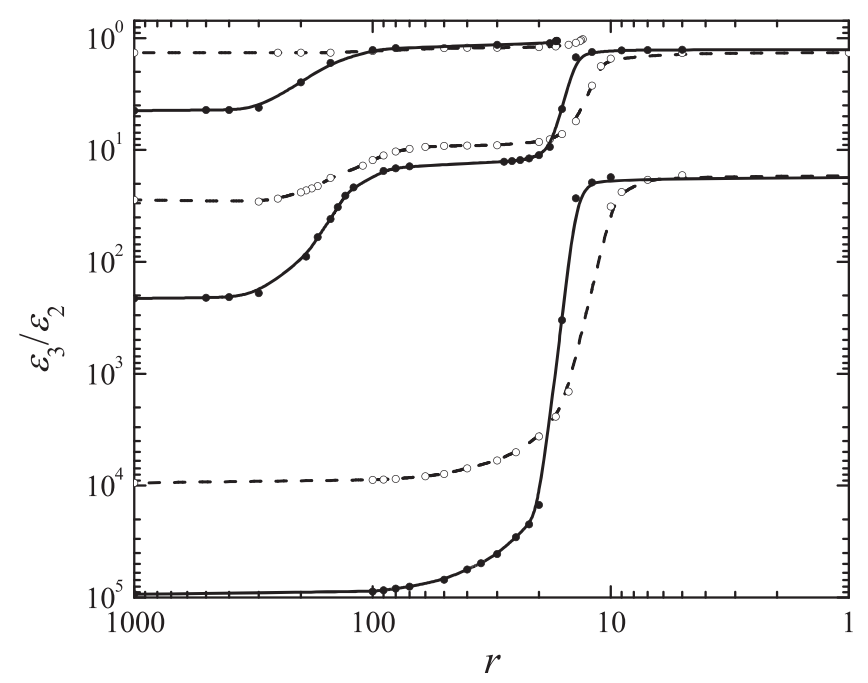

Figure 2. $\epsilon_{3} / \epsilon_{2}$ as a function of $r$, for $\epsilon_{2}^{3 \mathrm{D}}=10^{-7}$ (full circles) and $10^{-6}$ (empty circles). The solid and dashed lines are guides to the eye. As we approach the 2D limit $(r \rightarrow 0)$, higher excited states disappear and only the ground and first excited states remain. Note that the values of $r$ and $\epsilon_{3} / \epsilon_{2}$ increase from right to left and top to bottom respectively.

bound states. The points at which we calculated the energies are shown explicitly, while the curves are guides to the eye. For the largest $r=1000$, the energies were obtained from a pure 3D STM equation. The plot shows a very interesting dimensional crossover result. We have one sharp transition for the ground state and two for the first excited state, while the second excited state has two transitions before it disappears. This plot is reminiscient of the famous binding energy plot of Efimov trimers going from $a=\infty$ and into the three-body continuum [3]. The sharp jumps may also be interpreted as a sort of avoided crossing behaviour as $R$ changes. This is very similar to what is seen in other confined systems such as short-range interacting two-body systems in a harmonic trap [56]. The latter show similar jumps as function of the ratio of the interaction and confinement energy scales and have been related to the Zeldovich rearrangement effect seen for particles in strong magnetic fields [57, 58].

The jumps can be understood by considering the size of the trimer. It is given roughly by $\bar{r} \sim 1 / \sqrt{\epsilon_{3}}$. For $\epsilon_{2}=10^{-7}$, from the ground state plateau at $\epsilon_{3} / \epsilon_{2}=93330$ for $r=1000$ and first excited state plateau at $\epsilon_{3} / \epsilon_{2}=211.79$ also for $r=1000$. From the energies in the 3D limit, we predict that for $\bar{r}=217.29$ the ground state trimer has a size that matches the size of the squeezed dimension, $r$. For the first excited state the corresponding number is $\bar{r}=10.35$. Looking at figure 2 we see that these numbers match quite well with the values calculated numerically. This allows us to interpret the jumps as signaling that the $2 \mathrm{D}$ limit is reached first for the ground state and then for the first excited state. Due to the additional avoided crossing, we get the second jump for the first excited state after which it is forced to go to the only excited state that is present in the strict 2D limit for $r \rightarrow 0$. The same analysis can be made for $\epsilon_{2}=10^{-6}$ with $\bar{r}=10.27$ and $\bar{r}=188.98$, respectively, for the ground and first excited 
state. Varying $r$ from large to small values (left to right), the $3 \mathrm{D} \rightarrow 2 \mathrm{D}$ transition occurs for $r \sim 10$, where we have the disappearance of the higher (second in our case) excited states in order to reproduce the well known $2 \mathrm{D}$ results with two bound states proportional to $\epsilon_{3} / \epsilon_{2}=16.52$ and $\epsilon_{3} / \epsilon_{2}=1.27$ [38].

From the experimental point of view it may be difficult to keep the dimer energy, $E_{2}$, constant. However, the transitions observed in figure 2 will not disappear due to a variation of $\epsilon_{2}$ with $r$. A change in the dimer energy does not move the jumps significantly. Larger dimer energies will cause the 3D plateau to move to lower $\epsilon_{3} / \epsilon_{2}$ ratio and push the beginning of the transition to smaller $r$, thus making the transition region broader. In cases where there are four or more states in the spectrum, the higher states (second excited and above) will go to the continuum before one reaches the $2 \mathrm{D}$ limit. Whether they show two plateaus depends on whether they enter the spectrum above or below the values $\epsilon_{3} / \epsilon_{2}=16.52$ and $\epsilon_{3} / \epsilon_{2}=1.27$ in analogy to what we see in figure 2. In general, the jumps happen when a state is commensurate with the energy of the transverse squeezed dimension, while the associated plateaus are given by the $2 \mathrm{D}$ limit.

As an outlook we may consider a mass imbalanced system where the $2 \mathrm{D}$ limit can be much more rich with many bound states [59, 60]. This immediately implies that there could be more plateaus for these systems and that the sequence of jumps will be more involved but potentially even more interesting. We leave this issue for future studies.

\section{Acknowledgments}

The authors thank partial support from the Brazilian agencies FAPESP (2013/04093-3), CNPq and CAPES (88881.030363/ 2013-01), and by the Danish Council for Independent Research DFF Natural Sciences and the DFF Sapere Aude program. We thank M Valiente and J Levinsen for enlightening discussions.

\section{References}

[1] Drut J E and Nicholson A N 2013 J. Phys. G: Nucl. Part. Phys. 40043101

[2] Beane S R et al 2012 Phys. Rev. D 85054511 Aoki S et al 2012 Prog. Theor. Exp. Phys. 2012 01A105

[3] Efimov V 1970 Phys. Lett. B 33563 Efimov V 1981 Nucl. Phys. A 36245

[4] Lewenstein M et al 2007 Adv. Phys. 56243

[5] Bloch I, Dalibard J and Zwerger W 2008 Rev. Mod. Phys. 80885

[6] Zinner N T and Jensen A S 2013 J. Phys. G: Nucl. Part. Phys. 40053101

[7] Papp S B et al 2008 Phys. Rev. Lett. 101135301

[8] Pollack S E et al 2009 Phys. Rev. Lett. 102090402

[9] Navon N et al 2011 Phys. Rev. Lett. 107135301

[10] Wild R J et al 2012 Phys. Rev. Lett. 108145305

[11] Fletcher R J et al 2013 Phys. Rev. Lett. 111125303

[12] Rem B S et al 2013 Phys. Rev. Lett. 110163202

[13] Makotyn P et al 2014 Nat. Phys. 10116
[14] Ha L-I et al 2013 Phys. Rev. Lett. 110145302

[15] Makhalov V, Martiyanov K and Turlapov A 2014 Phys. Rev. Lett. 112045301

[16] Kraemer T et al 2006 Nature 440315

[17] Ottenstein T B, Lompe T, Kohnen M, Wenz A N and Jochim S 2008 Phys. Rev. Lett. 101203202

[18] Pollack S E, Dries D and Hulet R G 2009 Science 3261683

[19] Zaccanti M et al 2009 Nat. Phys. 5586

[20] Gross N, Shotan Z, Kokkelmans S and Khaykovich L 2009 Phys. Rev. Lett. 103163202

[21] Huckans J H, Williams J R, Hazlett E L, Stites R W and O'Hara K M 2009 Phys. Rev. Lett. 102165302

[22] Williams J R, Hazlett E L, Huckans J H, Stites R W, Zhang Y and O'Hara K M 2009 Phys. Rev. Lett. 103130404

[23] Lompe T, Ottenstein T B, Serwane F, Wenz A N, Zürn G and Jochim S 2010 Science 330940

[24] Gross N, Shotan Z, Kokkelmans S and Khaykovich L 2010 Phys. Rev. Lett. 105103203

[25] Lompe T, Ottenstein T B, Serwane F, Viering K, Wenz A N, Zürn G and Jochim S 2010 Phys. Rev. Lett. 105103201

[26] Nakajima S, Horikoshi M, Mukaiyama T, Naidon P and Ueda M 2010 Phys. Rev. Lett. 105023201

[27] Nakajima S, Horikoshi M, Mukaiyama T, Naidon P and Ueda M 2011 Phys. Rev. Lett. 106143201

[28] Berninger M et al 2011 Phys. Rev. Lett. 107120401

[29] Machtey O, Kessler D A and Khaykovich L 2012 Phys. Rev. Lett. 108130403

[30] Machtey O, Shotan Z, Gross N and Khaykovich L 2012 Phys. Rev. Lett. 108210406

[31] Knoop S, Borbely J S, Vassen W and Kokkelmans S J J M F 2012 Phys. Rev. A 86062705

[32] Dyke P, Pollack S E and Hulet R G 2013 Phys. Rev. A 88 023625

[33] Roy S et al 2013 Phys. Rev. Lett. 111053202

[34] Tung S-K et al 2014 arXiv:1402.5943

[35] Huang B, Sidorenkov L A, Grimm R and Hutson J M 2014 Phys. Rev. Lett. 112190401

[36] Pires R et al 2014 Phys. Rev. Lett. 112250404

[37] Chin C, Grimm R, Julienne P S and Tiesinga E 2010 Rev. Mod. Phys. 821225

[38] Bruch L W and Tjon J A 1979 Phys. Rev. A 19425

[39] Endres M G, Kaplan D B, Lee J-W and Nicholson A N 2013 Phys. Rev. A 87023615

[40] Rossi M, Salasnich L, Ancilotto F and Toigo F 2014 Phys. Rev. A $89041602(\mathrm{R})$

[41] Wilson K G 1975 Rev. Mod. Phys. 47773

[42] 't Hooft G 1973 Nucl. Phys. B 61455

[43] Nishida Y and Son D T 2010 The BCS-BEC Crossover and the Unitary Fermi Gas (Lecture Notes in Physics vol 836) ed W Zwerger (Berlin: Springer)

[44] Nishida Y and Tan S 2011 Few-Body Syst. 51191

[45] Levinsen J, Massignan P and Parish M M 2014 Phys. Rev. X 4 031020

[46] Gaunt A L et al 2013 Phys. Rev. Lett. 110200406

[47] Schmidutz T F et al 2014 Phys. Rev. Lett. 112040403

[48] Skorniakov G V and Ter-Martirosyan K A 1957 Sov. Phys.JETP 4648

[49] Yamashita M T et al 2013 Phys. Rev. A 87062702

[50] Castin Y and Werner F 2011 Phys. Rev. A 83063614

[51] Thomas L H 1935 Phys. Rev. 47903

[52] Adhikari S K and Frederico T 1995 Phys. Rev. Lett. 744572

[53] Adhikari S K, Delfino A, Frederico T, Goldman I D and Tomio L 1988 Phys. Rev. A 373666

[54] Petrov D S and Shlyapnikov G V 2001 Phys. Rev. A 64 012706

[55] Valiente M, Zinner N T and Mølmer K 2012 Phys. Rev. A 86 043616

[56] Busch T, Englert B-G, Rzążewski K and Wilkens M 1998 Found. Phys. 28549 
[57] Farrell A, MacDonald Z and van Zyl B 2012 J. Phys. A: Math. Theor. 45045303

[58] Zinner N T 2012 J. Phys. A: Math. Theor. 45205302

[59] Bellotti F F et al 2011 J. Phys. B: At. Mol. Opt. Phys. 44 205302
Bellotti F F et al 2012 Phys. Rev. A 85025601

Bellotti F F et al 2013 J. Phys. B: At. Mol. Opt. Phys. 46 055301

[60] Pricoupenko L and Pedri P 2010 Phys. Rev. A 82 033625 